Pak. j. sci. ind. res. Ser. B: biol. sci. 2020 63B(2) 119-126

\title{
Antibacterial Activity Evaluation of Psidium guajava L. (Myrtaceae) Crude Extracts Against Selected Bacterial Pathogens
}

\author{
Basel Saleh* and Ayman Al-Mariri \\ Department of Molecular Biology and Biotechnology, Atomic Energy Commission of Syria, P.O. Box 6091, \\ Damascus-Syria
}

(received March 13, 2018; revised August 27, 2018; accepted August 31, 2018)

\begin{abstract}
Methanol, ethanol, acetone, ethyl acetate and hot water extracts of different parts (leaves, seeds, fruits and twigs fractions) of Psidium guajava L. were investigated for their antibacterial activities against 8 bacterial isolates. Inhibitory effects of $P$. guajava L. extracts have been screened by discdiffusion method (Zone of inhibition, ZI), activity index (AI) and minimum inhibitory concentration (MIC) determination. Ciprofloxacin antibiotic was used as standard for P. guajava L. antimicrobial activity comparison. From the ZI, AI and MIC values, methanolic and hot water extracts of twigs $<1 \mathrm{~cm}$ diameter were the most potent against all tested micro-organisms by showing the highest ZI and AI value and the lowest MIC values. Whereas, no inhibitory activity was recorded for both seeds and fruits extracts using all tested solvents. These observations make this plant a potential source that can be used in management of bacterial infections. Moreover, methanolic and hot water extracts of twigs $<1 \mathrm{~cm}$ required further in depth study.
\end{abstract}

Keywords: Psidium guajava, antibacterial activity, minimum inhibitory concentration (MIC)

\section{Introduction}

The genus Psidium belongs to the family Myrtaceae, which is considered to have originated in tropical South America. Guava crops are grown in tropical and subtropical areas of the world especially Thailand, Pakistan, Egypt, Hawaii, Florida, Palestine, Syria and India, and others. The genus Psidium comprises approximately 150 species of small trees and shrubs in which only 20 species produce edible fruits and the rest are wild with inferior quality of fruits (Mani et al., 2011). The most commonly cultivated species of Psidium is $P$. guajava L. with common name "Guava".

Psidium guajava L. (Guava) is a perennial fruit tree that offers a great economic potential. Guava is one of the few medicinal plants which has been extensively investigated in terms of pharmacological activity of its phytochemical components (Farhana et al., 2017; Gitika1 and Kumar, 2016; Saleh et al., 2015; Mailoa et al., 2014; Taura et al., 2014; Ofodile et al., 2013; Biswas et al., 2013; Pandey and Shweta, 2012; Esimone et al., 2012; Elekwa et al., 2009; Nwinyi et al., 2008).

Guava is rich in antioxidants compounds, tannins, phenols, triterpenes, flavonoids, essential oils, saponins, carotenoids, lectins, vitamins (A, B, C \& G), fibre and fatty acids. The biology of these compounds indicated

*Author for correspondence; E-mail: bsaleh@aec.org.sy the great potent of their antibacterial activities. Guava fruit is higher in potassium, copper and manganese and about four times the amount of vitamin $\mathrm{C}$ as an orange (Hassimotto et al., 2005). Guava fruits are also a good source of pectin - a dietary fibre. The wood is hard and tough, used as posts for rural house buildings. In the Philippines, Syria, India, Pakistan, Nigeria and many other countries, guava fruit is freely eaten for its good taste and nutritional benefits.

The leaves and bark of $P$. guajava L. tree have a long history of medicinal uses that are still employed today (Nwinyi et al., 2008). Leaves and shoot of $P$. guajava exhibited their antibacterial activity against the both Gram-positive and Gram-negative bacteria such as $S$. aureus, Streptococcus mutans, Pseudomonas aeruginosa, Salmonella enteritidis, Bacillus cereus, Proteus vulgaris, Shigella dysenteriae and E. coli (Rattanachaikunsopon and Phumkhachorn, 2010). Moreover, Shruthi et al. (2013) reported the significant antibacterial activity of P. guajava L. and also its antifungal and antimalarial actions.

Mailoa et al. (2014) reported that flavonoids and tannins, out of the phenolic components, were present in majority in P. guajava L. leaves extracts and these compounds act as antimicrobial agent. It has achieved a very long history of traditional use for treating a wide range of diseases (Elekwa et al., 2009; Nwinyi et al., 2008). 
The long history of guava's use has led modern-day researchers to study guava extracts. Thereby, the current study aimed to investigate the inhibitory effect of methanol, ethanol, acetone, ethyl acetate and hot water extracts of leaves, seeds, fruits and twigs fractions of $P$. guajava L. against some bacterial pathogens using antibiotic ciprofloxacin as a standard reference.

\section{Materials and Methods}

Preparation of plant materials. Plant materials of fresh leaves, fruits, seeds and twigs $(<1 \mathrm{~cm}$ diameter and $>1 \mathrm{~cm}$ diameter) of the P. guajava L. were harvested from Lattakia-Syria. Samples collection was carried out in Spring with an annual rainfall ranging from 650 to $700 \mathrm{~mm}$. The twigs and leaves were dried under shade for one week, powdered by special electric mill and stored separately in polyethylene bags until needed for analysis.

Preparation of plant extracts. Different plant crude extracts were prepared by using different solvents such as methanol, ethanol, acetone, ethyl acetate and hot water. The extractions were performed in a Soxhlet apparatus successively as reported by Saleh et al. (2015). The extracts were dried using rotary evaporator at 40 ${ }^{\circ} \mathrm{C}$ under reduced pressure. All dried extracts were kept in air-tight bottles and stored at $4{ }^{\circ} \mathrm{C}$. The concentration of extract was considered $100 \mathrm{mg} / \mathrm{mL}$ (stock solution).

Micro-organisms and growth conditions. Eight pure clinical bacterial isolates (Staphylococcus aureus, Listeria monocytogenes, Bacillus cereus, Salmonella typhimurium, Escherichia coli O157, Proteus mirabilis, Pseudomonas aeruginosa and Klebsiella pneumoniae) were collected from the Microbiology and Immunology division, Department of Molecular Biology and Biotechnology, Atomic Energy Commission of Syria (AECS) in Damascus - Syria.

The cultures were activated in trypticase soy broth (TSB, Difco, BD, Spars, MD) at $37{ }^{\circ} \mathrm{C}$ for $24 \mathrm{~h}$. The medium was centrifuged $\left(1000 \mathrm{x} \mathrm{g} / 15 \mathrm{~min} / 4{ }^{\circ} \mathrm{C}\right)$ after growth and the pellets were resuspended in sterile phosphate-buffered saline (PBS). Prior to antimicrobial sensitivity test, a bacterial suspension was obtained from overnight cultures. The turbidity of each bacterial suspension was adjusted equivalent to a no. 0.5 McFarland standard and then inoculated on MuellerHinton agar (Oxoid, UK). The bacterial cultures standardized to approximately $10^{6} \mathrm{CFU} / \mathrm{mL}$ (Saleh et al., 2015). The exact counts were assessed retrospectively by viable counts on trypticase soy agar plates (TSA, Difco, BD, Spars, MD) at $37^{\circ} \mathrm{C}$ for $18 \mathrm{~h}$.

Antibacterial activity evaluation. The disc-diffusion method. Ciprofloxacin $(100 \mathrm{mg} / \mathrm{mL})$ antibiotic was used as standard for antimicrobial activity of bacteria. Filter paper discs (Whatman no.1) of $6 \mathrm{~mm}$ diameter were prepared and sterilized. The discs impregnated with $100 \mu \mathrm{L}$ of extract dilutions $(100 \mathrm{mg} / \mathrm{mL})$ were applied over each of the culture plates previously seeded with the $10^{6} \mathrm{CFU} / \mathrm{mL}$ cultures of bacteria. Bacterial cultures were then incubated at $37^{\circ} \mathrm{C}$ for $18 \mathrm{~h}$, while the paper discs impregnated with $20 \mu \mathrm{L}$ of extracts on each disc or solution of $10 \mathrm{mg} / \mathrm{mL}$ of ciprofloxacin were used as standard antimicrobials for comparison. Negative control was prepared using methanol or acetone (the highest concentration of solvent in the plant extract was tested). Diameter of inhibition zone (ZI in $\mathrm{mm}$ ) was measured after incubation at $37^{\circ} \mathrm{C}$ for $18-24 \mathrm{~h}$. For each extract, duplicate trials were conducted against each microorganism.

Activity index (A.I). Activity index (A.I) of P. guajava L. plant extracts was calculated as previously reported using the following formulae (Egharevba et al., 2010): Activity index (A.I) = Inhibition zone of sample / Inhibition zone of standard.

Minimum inhibitory concentration (MIC). Stock solutions of the above mentioned antibiotic was prepared according to manufacture. Determination of MIC by the microdilution broth method was carried out according to NCCLS approved standards. Microdilution broth susceptibility assay was used (Saleh et al., 2015). Three replicates of serial dilutions of extract $(100 \mathrm{mg} / \mathrm{mL})$ or of antibiotic $(128 \mu \mathrm{g} / \mathrm{mL})$ were prepared in TSB medium in 96-well microtiter plates. One hundred microliters of freshly grown cultures containing $10^{6} \mathrm{CFU} / \mathrm{mL}$ in TSB were added to each well. Positive control was achieved with the same conditions but without extract or antibiotics, negative control was also made with the same conditions but without adding the bacteria. The MIC was defined as the lowest concentration of each antimicrobial agent that inhibited visible growth of the tested isolate was recorded and interpreted as the MIC.

Statistical analysis. Results were expressed as mean of three replicates/treatment. Data were subjected to one way analysis of variance (ANOVA) for determining level of differences among variables. Differences between means were tested for significance by Fisher's Least Significant Difference (PLSD) test. The means were separated by using the student's t-test with $\mathrm{P} \leq$ 0.05 was considered to be significant. 


\section{Results and Discussion}

Zone of inhibitions (ZI). Inhibitory effect (ZI) of extracts of leaves (LE), seeds (SE), fruits (FE) and twigs (TE) of $P$. guajava L. against 8 bacterial isolates are presented Table 1. Analysis of variance using PLSD test at $\mathrm{P} \leq 0.05$ revealed statistically significant differences in ZI against all tested bacterial isolates among all examined solvents.

Inhibitory effect of P. guajava extracts varied according to the plant fractions, bacterial isolates and the solvents. Methanol extract, among the different solvents used, was the most potent against the eight tested bacterial isolates in this investigation. The zone of inhibition (ZI) observed for this extract ranged between $9-18 \mathrm{~mm}$. The highest $\mathrm{ZI}$ value was recorded to be $18 \mathrm{~mm}$ for $\mathrm{LE}$ and $\mathrm{TE}<1$ against $S$. typhimurium and LE against $P$. mirabilis isolate. Whereas, the lowest value was found to be $9 \mathrm{~mm}$ for TE $>1$ against $K$. pneumoniae. The ethanol, acetone, ethyl acetate and hot water extracts were active against only S. typhimurium isolate (Table 1).

Previously, Abdelrahim et al. (2002) reported that $P$. guajava aqueous and methanolic bark extracts exhibited antibacterial activity.

In our case study, ethanolic $P$. guajava extracts were non active against all tested isolates except $S$. typhimurium. Whereas, This value was ranged between 19.4-21.9 mm against $L$. monocytogenes strain and between 10.7-15.3 $\mathrm{mm}$ against $S$. aureus strain with ethanolic $P$. guajava LE (Mahfuzul Hoque et al., 2007). While, this value was $25,28,26$ and $25 \mathrm{~mm}$ with $10,25,50$ and $100 \%$ methanolic $P$. guajava leaf extracts, respectively against S. typhi isolate (Ahmed and Yagoub, 2007).

Dhiman et al. (2011) reported antibacterial effect of methanolic $P$. guajava leaf extracts $(1,3,5,10$ and 20 $\mu \mathrm{g} / \mathrm{mL}$ ) against 3 bacterial pathogens. Their study revealed that the highest ZI was recorded to be 10.6 $\mathrm{mm}$ against $E$. coli, $9.6 \mathrm{~mm}$ against $S$. aureus and 12.6 $\mathrm{mm}$ against $B$. subtilis at the highest concentration (20 $\mu \mathrm{g} / \mathrm{mL}$ ). in our case study this value was recorded to be 13,15 and $16 \mathrm{~mm}$ with methanolic $\mathrm{TE}<1 \mathrm{~cm}$ and to be 11,13 and $11 \mathrm{~mm}$ with methanolic TE $>1 \mathrm{~cm}$ against $P$. aeruginosa, $S$. aureus and E. coli isolates, respectively, whereas, this value was $28.5,20$ and 22.5 $\mathrm{mm}$ against $P$. aeruginosa, $S$. aureus and $E$. coli isolates, respectively using methanolic stem $P$. guajava extracts (Pandey and Shweta, 2012).

In our case study, ZI was recorded to be 13,16 and 15 $\mathrm{mm}$ against $S$. aureus isolate with methanolic TE $>1$ $\mathrm{cm}, \mathrm{LE}$ and $\mathrm{TE}<1 \mathrm{~cm}$ extracts, respectively. Whereas it ranged between $5-15 \mathrm{~mm}(20 \mathrm{mg} / \mathrm{mL}$ extract $)$ and between $6-20 \mathrm{~mm}(40 \mathrm{mg} / \mathrm{mL}$ extract $)$ against 8 methicillin-resistant $S$. aureus (MRSA) isolates using P. guajava stem bark methanolic extracts (Esimone et al., 2012).

In the current study, this value was recorded to be 17 and $16 \mathrm{~mm}$ against $B$. cereus and $S$. aureus, respectively using methanolic P. guajava LE extract. Whereas, ethanolic one had no activity against all tested isolates except $S$. typhimurium isolate. While, this value was 8.27 and $12.3 \mathrm{~mm}$ with methanolic P. guajava leaf extracts and to be 6.11 and $11.0 \mathrm{~mm}$ with ehanolic ones against $B$. cereus and $S$. aureus, respectively (Biswas et al., 2013).

Moreover, Mailoa et al. (2014) reported the antimicrobial activity of extracted tannins (different concentrations) from P. guajava L. against 3 bacterial and 2 fungal pathogens. This investigation suggested that, antimicrobial activity increased as tannins concentration increased. In this respect, for bacteria ZI at 30\% ranged between $12 \mathrm{~mm}$ (E. coli) and $14.5 \mathrm{~mm}$ (P. aeruginosa); while this value at the same tannins concentration varied between $9 \mathrm{~mm}$ (C. albicans) and $9.5 \mathrm{~mm}$ (A. niger) for fungal pathogens. Saleh et al. (2015) reported antimicrobial effects of methanol, ethanol, acetone, ethyl acetate and hot water (leaves and twigs fractions) P. guajava L. crude extracts against three bacterial and two fungal pathogens. they reported that hot water twigs $<1 \mathrm{~cm}$ diameter extract displayed the highest ZI of $20 \mathrm{~mm}$ with hot water twigs $<1 \mathrm{~cm}$ against $C$. albicans fungi. Whereas, for bacteria, the highest ZI was recorded to be $19 \mathrm{~mm}$ with methanolic LE against Acinetobacter baumannii bacterial isolate.

In the current investigation ZI value was recorded to be 12,13 and $16 \mathrm{~mm}$ against $K$. pneumoniae, $P$. aeruginosa and $S$. aureus isolates, respectively using methanolic LE. Whereas, this value was 8,10 and 10 mm with $480 \mu \mathrm{g} /$ disc $P$. guajava leaves at $45^{\circ} \mathrm{C}$ against Klebsiella spp, Pseudomonas spp and Staphylococcus spp, respectively (Taura et al., 2014). While, it was 21, 23, 20, 18, 22, 14 and $17 \mathrm{~mm}$ against Bacillus subtilis, Micrococcus luteus, S. aureus, Streptococcus sp., E. coli, $P$. aeruginosa and $S$. typhimurium, respectively using P. guajava L. leaves extracts (methanol, ethanol and aqueous extracts (Gitikal and Kumar 2016). Whereas, it was $13,16,18,19$ and $20 \mathrm{~mm}$ with methanolic leaf extracts and to be 22, 15, 19, 23 and 
Table 1. Antibacterial activity of the P. guajava L. extracts using disc-diffusion test

\begin{tabular}{|c|c|c|c|c|c|}
\hline \multirow[b]{2}{*}{ Solvent } & \multirow[b]{2}{*}{ Tested micro-organisms } & \multicolumn{3}{|c|}{ Zone of inhibition (mm) } & \multirow[b]{2}{*}{ Control } \\
\hline & & LE & $\mathrm{TE}<1 \mathrm{~cm}$ & $\mathrm{TE}>1 \mathrm{~cm}$ & \\
\hline \multirow{8}{*}{ Methanol } & S. aureus & $16 \pm 0.2^{\mathrm{Aa}}$ & $15 \pm 0.09^{\mathrm{Ba}}$ & $13 \pm 0.07^{\mathrm{Ab}}$ & $24 \pm 0.2$ \\
\hline & L. monocytogenes & $14 \pm 0.19^{\mathrm{Bb}}$ & $16 \pm 0.24^{\mathrm{Aa}}$ & $11 \pm 0.09^{\mathrm{Ac}}$ & $19 \pm 0.12$ \\
\hline & B. cereus & $17 \pm 0.28^{\mathrm{Aa}}$ & $15 \pm 0.19^{\mathrm{Bb}}$ & $12 \pm 0.12^{\mathrm{Ac}}$ & $21 \pm 0.07$ \\
\hline & S. typhimurium & $18 \pm 0.0^{\mathrm{Aa}}$ & $18 \pm 0.0^{\mathrm{Aa}}$ & $13 \pm 0.0^{\mathrm{Ab}}$ & $34 \pm 0.2$ \\
\hline & E. coli $\mathrm{O}: 157$ & $17 \pm 0.03^{\mathrm{Aa}}$ & $16 \pm 0.1^{\mathrm{Aa}}$ & $11 \pm 0.06^{\mathrm{Ab}}$ & $27 \pm 0.15$ \\
\hline & P. mirabilis & $18 \pm 0.17^{\mathrm{Aa}}$ & $14 \pm 0.26^{\mathrm{Bb}}$ & $13 \pm 0.24^{\mathrm{Ab}}$ & $19 \pm 0.25$ \\
\hline & P. aeruginosa & $13 \pm 0.08^{\mathrm{Bb}}$ & $13 \pm 0.17^{\mathrm{Ba}}$ & $11 \pm 0.13^{\mathrm{Ab}}$ & $15 \pm 0.06$ \\
\hline & K. pneumoniae & $12 \pm 0.11^{\mathrm{Bb}}$ & $13 \pm 0.29^{\mathrm{Ba}}$ & $9 \pm 0.08^{\mathrm{Bb}}$ & $18 \pm 0.32$ \\
\hline \multirow{8}{*}{ Ethanol } & S. aureus & na & na & na & $24 \pm 0.2$ \\
\hline & L. monocytogenes & na & na & na & $19 \pm 0.12$ \\
\hline & B. cereus & na & na & na & $21 \pm 0.07$ \\
\hline & S. typhimurium & $11 \pm 0.0^{\mathrm{Cb}}$ & $16 \pm 0.0^{\mathrm{Aa}}$ & $9 \pm 0.0^{\mathrm{Bc}}$ & $34 \pm 0.2$ \\
\hline & E. coli $\mathrm{O}: 157$ & na & na & na & $27 \pm 0.15$ \\
\hline & P. mirabilis & na & na & na & $19 \pm 0.25$ \\
\hline & P. aeruginosa & na & na & na & $15 \pm 0.06$ \\
\hline & K. pneumoniae & na & na & na & $18 \pm 0.32$ \\
\hline \multirow{8}{*}{ Acetone } & S. aureus & na & na & na & $24 \pm 0.2$ \\
\hline & L. monocytogenes & na & na & na & $19 \pm 0.12$ \\
\hline & B. cereus & na & na & na & $21 \pm 0.07$ \\
\hline & S. typhimurium & $12 \pm 0.0^{\mathrm{Bb}}$ & $14 \pm 0.0^{\mathrm{Ba}}$ & $11 \pm 0.0^{\mathrm{Ab}}$ & $34 \pm 0.2$ \\
\hline & E. coli $\mathrm{O}: 157$ & na & na & na & $27 \pm 0.15$ \\
\hline & P. mirabilis & na & na & na & $19 \pm 0.25$ \\
\hline & P. aeruginosa & na & na & na & $15 \pm 0.06$ \\
\hline & K. pneumoniae & na & na & na & $18 \pm 0.32$ \\
\hline \multirow{8}{*}{ Ethyl acetate } & S. aureus & na & na & na & $24 \pm 0.2$ \\
\hline & L. monocytogenes & na & na & na & $19 \pm 0.12$ \\
\hline & B. cereus & na & na & na & $21 \pm 0.07$ \\
\hline & S. typhimurium & $17 \pm 0.11^{\mathrm{Aa}}$ & na & na & $34 \pm 0.2$ \\
\hline & E. coli $\mathrm{O}: 157$ & na & na & na & $27 \pm 0.15$ \\
\hline & P. mirabilis & na & na & na & $19 \pm 0.25$ \\
\hline & P. aeruginosa & na & na & na & $15 \pm 0.06$ \\
\hline & K. pneumoniae & na & na & na & $18 \pm 0.32$ \\
\hline \multirow{8}{*}{ Hot water } & S. aureus & na & na & na & $24 \pm 0.2$ \\
\hline & L. monocytogenes & na & na & na & $19 \pm 0.12$ \\
\hline & B. cereus & na & na & na & $21 \pm 0.07$ \\
\hline & S. typhimurium & $13 \pm 0.0^{\mathrm{Ba}}$ & $13 \pm 0.0^{\mathrm{Ba}}$ & $12 \pm 0.0^{\mathrm{Aa}}$ & $34 \pm 0.2$ \\
\hline & E. coli $\mathrm{O}: 157$ & na & na & na & $27 \pm 0.15$ \\
\hline & P. mirabilis & na & na & na & $19 \pm 0.25$ \\
\hline & P. aeruginosa & na & na & na & $15 \pm 0.06$ \\
\hline & K. pneumoniae & na & na & na & $18 \pm 0.32$ \\
\hline
\end{tabular}

LE= Leaf extracts; TE= Twig extracts; na= no activity; Figures sharing same capital letter (column) and lowercase letter (row) are not significantly different at $\mathrm{P}=0.05$ probability by Fisher's PLSD test. LSD 0.05 solvent 2.200, plant part 1.362 .

$18 \mathrm{~mm}$ with ethanolic leaf extracts against $P$. aeruginosa, K. pneumonia, Streptococcus pneumonia, S. aureus and E. coli pathogens, respectively, while, for bark once, it was recorded to be $20,14,13,23$ and $19 \mathrm{~mm}$ with methanolic bark extracts and to be $19,18,16,18$ and $19 \mathrm{~mm}$ with ethanolic bark extracts against $P$. aeruginosa, K. pneumonia, Streptococcus pneumonia,
S. aureus and E. coli pathogens, respectively (Ifeanyichukwu et al., 2015).

Recently, Farhana et al. (2017) reported that this value was $12.67,9.83,13.5,17$ and $8.67 \mathrm{~mm}$ against $S$. aureus, S. typhi, E. coli, B. cereus and Shigella sonnei isolates, respectively using $100 \%$ fresh guava extracts. 
Activity index (A.I). Activity index (A.I) was also estimated to investigate $P$. guajava L. inhibitory effect (Table 2). Data presented herein showed that this parameter varied according to plant fraction, examined solvent and tested microorganism (Table 2). Statistical variance analysis revealed that the effect of plant parts extracts expressed as A.I on all tested bacterial isolates among all examined solvents were significantly $(P \leq$ 0.05 ) different (Table 2).

It was noticed that, ethanol, acetone, ethyl acetate and hot water were active against only $S$. typhimurium with A.I ranged between $0.3-0.5,0.3-0.4,0.5$ and 0.4 for the previous tested solvents, respectively. Whereas, for

Table 2. Activity index (A.I) of the P. guajava L. extracts against tested isolates

\begin{tabular}{|c|c|c|c|c|}
\hline \multirow[b]{2}{*}{ Solvent } & \multicolumn{3}{|c|}{ Activity index (A.I) } & \multirow[b]{2}{*}{$\mathrm{TE}>1 \mathrm{~cm}$} \\
\hline & Tested micro-organisms & LE & $\mathrm{TE}<1 \mathrm{~cm}$ & \\
\hline \multirow{8}{*}{ Methanol } & S. aureus & $0.7 \pm 0.01^{\mathrm{Ca}}$ & $0.6 \pm 0.01^{\mathrm{Db}}$ & $0.5 \pm 0.01^{\mathrm{Ec}}$ \\
\hline & L. monocytogenes & $0.7 \pm 0.01^{\mathrm{Cb}}$ & $0.8 \pm 0.01^{\mathrm{Ba}}$ & $0.6 \pm 0.01^{\mathrm{Dc}}$ \\
\hline & B. cereus & $0.8 \pm 0.01^{\mathrm{Ba}}$ & $0.7 \pm 0.01^{\mathrm{Cb}}$ & $0.6 \pm 0.01^{\mathrm{Dc}}$ \\
\hline & S. typhimurium & $0.5 \pm 0.01^{\mathrm{Ea}}$ & $0.5 \pm 0.01^{\mathrm{Eb}}$ & $0.4 \pm 0.01^{\mathrm{Fb}}$ \\
\hline & E. coli $O: 157$ & $0.6 \pm 0.01^{\mathrm{Da}}$ & $0.5 \pm 0.01^{\mathrm{Eb}}$ & $0.4 \pm 0.01^{\mathrm{Fc}}$ \\
\hline & P. mirabilis & $0.9 \pm 0.02^{\mathrm{Aa}}$ & $0.7 \pm 0.01^{\mathrm{Cb}}$ & $0.7 \pm 0.01^{\mathrm{Cb}}$ \\
\hline & P. aeruginosa & $0.9 \pm 0.02^{\mathrm{Aa}}$ & $0.9 \pm 0.02^{\mathrm{Aa}}$ & $0.7 \pm 0.01^{\mathrm{Cb}}$ \\
\hline & K. pneumoniae & $0.7 \pm 0.01^{\mathrm{Ca}}$ & $0.7 \pm 0.01^{\mathrm{Ca}}$ & $0.5 \pm 0.01^{\mathrm{Eb}}$ \\
\hline \multirow{8}{*}{ Ethanol } & S. aureus & - & - & - \\
\hline & L. monocytogenes & - & - & - \\
\hline & B. cereus & - & - & - \\
\hline & S. typhimurium & $0.3 \pm 0.00^{\mathrm{Gb}}$ & $0.5 \pm 0.01^{\mathrm{Ea}}$ & $0.3 \pm 0.00^{\mathrm{Gb}}$ \\
\hline & E. coli $O: 157$ & - & - & -- \\
\hline & P. mirabilis & - & - & - \\
\hline & P. aeruginosa & - & - & - \\
\hline & K. pneumoniae & - & -- & - \\
\hline \multirow{8}{*}{ Acetone } & S. aureus & - & -- & -- \\
\hline & L. monocytogenes & - & -- & -- \\
\hline & B. cereus & - & -- & - \\
\hline & S. typhimurium & $0.4 \pm 0.01^{\mathrm{Fa}}$ & $0.4 \pm 0.01^{\mathrm{Fa}}$ & $0.3 \pm 0.00^{\mathrm{Gb}}$ \\
\hline & E. coli $O: 157$ & - & - & - \\
\hline & P. mirabilis & - & - & - \\
\hline & P. aeruginosa & - & - & - \\
\hline & K. pneumoniae & - & - & - \\
\hline \multirow{8}{*}{ Ethyl acetate } & S. aureus & - & - & - \\
\hline & L. monocytogenes & - & - & - \\
\hline & B. cereus & - & - & - \\
\hline & S. typhimurium & $0.5 \pm 0.01^{\mathrm{Ea}}$ & - & - \\
\hline & E. coli $O: 157$ & - & - & - \\
\hline & P. mirabilis & - & - & - \\
\hline & P. aeruginosa & - & - & - \\
\hline & K. pneumoniae & - & - & - \\
\hline \multirow{8}{*}{ Hot water } & S. aureus & - & - & - \\
\hline & L. monocytogenes & - & - & - \\
\hline & B. cereus & - & - & - \\
\hline & S. typhimurium & $0.4 \pm 0.01^{\mathrm{Fa}}$ & $0.4 \pm 0.01^{\mathrm{Fa}}$ & $0.4 \pm 0.01^{\mathrm{Fa}}$ \\
\hline & E. coli $O: 157$ & - & - & - \\
\hline & P. mirabilis & - & - & - \\
\hline & P. aeruginosa & - & - & - \\
\hline & K. pneumoniae & - & - & - \\
\hline
\end{tabular}

$\mathrm{LE}=$ Leaf extracts; TE $=$ Twig extracts; no activity Figures sharing same capital letter (column) and lowercase letter (row) are not significantly different at $\mathrm{P}=0.05$ probability by Fisher's PLSD test. LSD 0.05 solvent 0.073 , plant part 0.057 . 
methanol one, this value ranged between $0.4-0.9$. In this respect the highest value was recorded to be 0.9 for both $\mathrm{LE}$ and $\mathrm{TE}<1$ against $P$. aeruginosa isolate and LE against $P$. mirabilis. Previously, Egharevba et al. (2010) reported lower values for this parameter $(0$, $0.63,0.63$ and 0 ) using $70 \%$ methanol, methanol, erythromycin and hexane $P$. guajava LE against $C$. albicans. Whereas, Saleh et al. (2015) reported that hot water TE $<1 \mathrm{~cm}$ exhibited the highest A.I against $C$. albicans fungi (2). While, for bacterial isolates, LE ethyl acetate exhibited the highest A.I against $S$. pneumoniae (1.7), followed by LE hot water (1.6) and $\mathrm{TE}<1 \mathrm{~cm}$ (1.6) against the same isolate. Our data were in coherent with findings of Saleh et al. (2015), who

Table 3. Minimum inhibitory concentration (MIC) of the P. guajava L. extracts against tested isolates

\begin{tabular}{|c|c|c|c|c|}
\hline \multirow[b]{2}{*}{ Solvent } & \multicolumn{3}{|c|}{ Minimum inhibitory concentration values $(\mathrm{mg} / \mathrm{mL})$} & \multirow[b]{2}{*}{$\mathrm{TE}>1 \mathrm{~cm}$} \\
\hline & Tested microorganisms & LE & $\mathrm{TE}<1 \mathrm{~cm}$ & \\
\hline \multirow{8}{*}{ Methanol } & S. aureus & $10.3 \pm 0.58^{\mathrm{Da}}$ & $8.3 \pm 0.58^{\mathrm{Fa}}$ & $16.7 \pm 1.15^{\mathrm{Fa}}$ \\
\hline & L. monocytogenes & $8.3 \pm 0.58^{\mathrm{Da}}$ & $7.3 \pm 0.58^{\mathrm{Fa}}$ & $12.5 \pm 2.52^{\mathrm{Ga}}$ \\
\hline & B. cereus & $10.3 \pm 0.58^{\mathrm{Da}}$ & $5.0 \pm 0.00^{\mathrm{Fa}}$ & $16.7 \pm 1.15^{\mathrm{Fa}}$ \\
\hline & S. typhimurium & $5.0 \pm 0.00^{\mathrm{Db}}$ & $5.0 \pm 0.00^{\mathrm{Fb}}$ & $33.3 \pm 0.58^{\mathrm{Fa}}$ \\
\hline & E. coli $O: 157$ & $7.3 \pm 0.58^{\mathrm{Db}}$ & $7.3 \pm 0.58^{\mathrm{Fb}}$ & $41.7 \pm 1.53^{\mathrm{Ea}}$ \\
\hline & P. mirabilis & $5.0 \pm 0.00^{\mathrm{Db}}$ & $5.0 \pm 0.00^{\mathrm{Fb}}$ & $29.3 \pm 0.58^{\mathrm{Fa}}$ \\
\hline & P. aeruginosa & $8.3 \pm 0.58^{\mathrm{Db}}$ & $8.3 \pm 0.58^{\mathrm{Fb}}$ & $20.7 \pm 1.15^{\mathrm{Fa}}$ \\
\hline & K. pneumoniae & $14.3 \pm 3.06^{\mathrm{Db}}$ & $9.3 \pm 0.58^{\mathrm{Fb}}$ & $29.3 \pm 0.58^{\mathrm{Fa}}$ \\
\hline \multirow{8}{*}{ Ethanol } & S. aureus & $16.7 \pm 1.15^{\mathrm{Db}}$ & $7.3 \pm 0.58^{\mathrm{Fb}}$ & $33.3 \pm 0.58^{\mathrm{Fa}}$ \\
\hline & L. monocytogenes & $16.7 \pm 1.15^{\mathrm{Da}}$ & $5.0 \pm 0.00^{\mathrm{Fb}}$ & $25.0 \pm 0.00^{\mathrm{Fa}}$ \\
\hline & B. cereus & $20.7 \pm 1.52^{\mathrm{Db}}$ & $4.0 \pm 0.00^{\mathrm{Fc}}$ & $33.3 \pm 0.58^{\mathrm{Fa}}$ \\
\hline & S. typhimurium & $20.7 \pm 1.52^{\mathrm{Db}}$ & $7.3 \pm 0.58^{\mathrm{Fc}}$ & $58.3 \pm 3.06^{\mathrm{Ea}}$ \\
\hline & E. coli $O: 157$ & $25.0 \pm 0.00^{\mathrm{Cb}}$ & $8.3 \pm 0.58^{\mathrm{Fc}}$ & $50.0 \pm 0.00^{\mathrm{Ea}}$ \\
\hline & P. mirabilis & $20.7 \pm 1.52^{\mathrm{Db}}$ & $10.3 \pm 0.58^{\mathrm{Fb}}$ & $58.3 \pm 3.06^{\mathrm{Ea}}$ \\
\hline & P. aeruginosa & $20.7 \pm 1.52^{\mathrm{Db}}$ & $10.3 \pm 0.58^{\mathrm{Fb}}$ & $50.0 \pm 0.00^{\mathrm{Ea}}$ \\
\hline & K. pneumoniae & $29.3 \pm 0.58^{\mathrm{Ca}}$ & $14.3 \pm 3.06^{\mathrm{Fb}}$ & $41.7 \pm 1.53^{\mathrm{Ea}}$ \\
\hline \multirow{8}{*}{ Acetone } & S. aureus & $29.3 \pm 0.58^{\mathrm{Ca}}$ & $8.3 \pm 0.58^{\mathrm{Fb}}$ & $20.7 \pm 1.15^{\mathrm{Fa}}$ \\
\hline & L. monocytogenes & $14.3 \pm 3.06^{\mathrm{Da}}$ & $7.3 \pm 0.58^{\mathrm{Fa}}$ & $14.3 \pm 3.06^{\mathrm{Ga}}$ \\
\hline & B. cereus & $12.3 \pm 0.58^{\mathrm{Da}}$ & $8.3 \pm 0.58^{\mathrm{Fa}}$ & $10.3 \pm 0.58^{\mathrm{Ga}}$ \\
\hline & S. typhimurium & $10.3 \pm 0.58^{\mathrm{Db}}$ & $8.3 \pm 0.58^{\mathrm{Fb}}$ & $29.3 \pm 0.58^{\mathrm{Fa}}$ \\
\hline & E. coli $O: 157$ & $14.3 \pm 3.06^{\mathrm{Db}}$ & $7.3 \pm 0.58^{\mathrm{Fb}}$ & $41.7 \pm 1.53^{\mathrm{Ea}}$ \\
\hline & P. mirabilis & $10.3 \pm 0.58^{\mathrm{Db}}$ & $10.3 \pm 0.58^{\mathrm{Fb}}$ & $33.3 \pm 0.58^{\mathrm{Fa}}$ \\
\hline & P. aeruginosa & $8.3 \pm 0.58^{\mathrm{Db}}$ & $10.3 \pm 0.58^{\mathrm{Fb}}$ & $33.3 \pm 0.58^{\mathrm{Fa}}$ \\
\hline & K. pneumoniae & $10.3 \pm 0.58^{\mathrm{Db}}$ & $8.3 \pm 0.58^{\mathrm{Fb}}$ & $41.7 \pm 1.53^{\mathrm{Ea}}$ \\
\hline \multirow{8}{*}{ Ethyl acetate } & S. aureus & $41.7 \pm 1.53^{\mathrm{Ca}}$ & $37.3 \pm 3.06^{\mathrm{Eb}}$ & $58.3 \pm 3.06^{\mathrm{Ea}}$ \\
\hline & L. monocytogenes & $66.7 \pm 5.8^{\mathrm{Ba}}$ & $54.3 \pm 1.15^{\mathrm{Db}}$ & $50.0 \pm 0.00^{\mathrm{Eb}}$ \\
\hline & B. cereus & $116.7 \pm 15.28^{\mathrm{Aa}}$ & $66.7 \pm 5.8^{\mathrm{Dc}}$ & $83.3 \pm 2.89^{\mathrm{Db}}$ \\
\hline & S. typhimurium & $83.3 \pm 2.89^{\mathrm{Bc}}$ & $116.7 \pm 15.28^{\mathrm{Cb}}$ & $233.3 \pm 15.28^{\mathrm{Ba}}$ \\
\hline & E. coli $O: 157$ & $100.0 \pm 0.00^{\mathrm{Ab}}$ & $116.7 \pm 15.28^{\mathrm{Ca}}$ & $116.7 \pm 15.28^{\mathrm{Ca}}$ \\
\hline & P. mirabilis & $116.7 \pm 15.28^{\mathrm{Ac}}$ & $133.3 \pm 11.55^{\mathrm{Bb}}$ & $266.7 \pm 28.87^{\mathrm{Aa}}$ \\
\hline & P. aeruginosa & $116.7 \pm 15.28^{\mathrm{Ac}}$ & $150.0 \pm 0.00^{\mathrm{Bb}}$ & $266.7 \pm 28.87^{\mathrm{Aa}}$ \\
\hline & K. pneumoniae & $83.3 \pm 2.89^{\mathrm{Bb}}$ & $233.3 \pm 15.28^{\mathrm{Aa}}$ & $233.3 \pm 15.28^{\mathrm{Ba}}$ \\
\hline \multirow{8}{*}{ Hot water } & S. aureus & $7.0 \pm 0.00^{\mathrm{Db}}$ & $4.0 \pm 0.00^{\mathrm{Fb}}$ & $25.0 \pm 0.00^{\mathrm{Fa}}$ \\
\hline & L. monocytogenes & $5.0 \pm 0.00^{\mathrm{Da}}$ & $4.0 \pm 0.00^{\mathrm{Fa}}$ & $10.3 \pm 0.58^{\mathrm{Ga}}$ \\
\hline & B. cereus & $6.0 \pm 0.00^{\mathrm{Da}}$ & $5.0 \pm 0.00 \mathrm{Fa}$ & $8.3 \pm 0.58^{\mathrm{Ga}}$ \\
\hline & S. typhimurium & $13.3 \pm 0.58^{\mathrm{Db}}$ & $4.0 \pm 0.00^{\mathrm{Fb}}$ & $50.0 \pm 0.00^{\mathrm{Ea}}$ \\
\hline & E. coli $O: 157$ & $14.3 \pm 0.58^{\mathrm{Db}}$ & $6.3 \pm 0.58^{\mathrm{Fb}}$ & $41.7 \pm 1.53^{\mathrm{Ea}}$ \\
\hline & P. mirabilis & $13.3 \pm 0.58^{\mathrm{Db}}$ & $4.0 \pm 0.00^{\mathrm{Fb}}$ & $50.0 \pm 0.00^{\mathrm{Ea}}$ \\
\hline & P. aeruginosa & $14.3 \pm 0.58^{\mathrm{Db}}$ & $8.3 \pm 0.58^{\mathrm{Fb}}$ & $66.7 \pm 5.8^{\mathrm{Da}}$ \\
\hline & K. pneumoniae & $16.7 \pm 1.15^{\mathrm{Db}}$ & $13.3 \pm 0.58^{\mathrm{Fb}}$ & $83.3 \pm 2.89^{\mathrm{Da}}$ \\
\hline
\end{tabular}

$\mathrm{LE}=$ Leaf extracts; TE= Twig extracts; Figures sharing same capital letter (column) and lowercase letter (row) are not significantly different at $\mathrm{P}=0.05$ probability by Fisher's PLSD test. LSD 0.05 solvent 18.089 , plant part 12.202. 
reported that $\mathrm{TE}<1 \mathrm{~cm}$ exhibited the highest $\mathrm{A}$.I value against bacterial isolates.

Minimum inhibitory concentration (MIC). MIC was also estimated (Table 3 ) to investigate inhibitory activity of P. guajava extracts. In this respect, MIC value of methanolic extract ranged between $5-116.7 \mathrm{mg} / \mathrm{mL}$, while, it was $4-233.3 \mathrm{mg} / \mathrm{mL}$ for $\mathrm{TE}<1$. Whereas, it was varied between $8.3-266.7 \mathrm{mg} / \mathrm{mL}$ for $\mathrm{TE}>1$ (Table 3). It worth noting that, the hot water TE $<1$ extract exhibited the lowest MIC value $(4-13.3 \mathrm{mg} / \mathrm{mL})$ (Table 3). Analysis of variance using PLSD test at $\mathrm{P} \leq$ 0.05 revealed statistically significant differences in MIC against all tested bacterial isolates among all examined solvents (Table 3). In this regards, MIC estimated values for hot water TE $<1$ extract were as follows: $4 \mathrm{mg} / \mathrm{ml}$ against $S$. aureus, L. monocytogeneses, $S$. typhimurium and $P$. mirabilis $<5 \mathrm{mg} / \mathrm{mL}$ against $B$. cereus $<6.3$ $\mathrm{mg} / \mathrm{mL}$ against $E$. coli $\mathrm{O}: 157<8.3 \mathrm{mg} / \mathrm{mL}$ against $P$. aeruginosa $<13.3 \mathrm{mg} / \mathrm{mL}$ against $K$. pneumoniae isolate.

In the current study, ethanolic extract was non active against all tested isolates except against $S$. typhimurium. Whereas, the lowest lowest MIC value was recorded to be $0.1 \mathrm{mg} / \mathrm{mL}$ with ethanol $P$. guajava extracts against each of L. monocytogenes, S. aureus and Vibrio parahaemolyticus isolates (Mahfuzul Hoque et al., 2007). Moreover, MIC value was recorded to be 10.3 and $7.3 \mathrm{mg} / \mathrm{mL}$ against $S$. aureus with methanolic and hot water LE extracts, respectively. Other study revealed that MIC value was ranged between $625 \mu \mathrm{g} / \mathrm{mL}-7.5$ $\mathrm{mg} / \mathrm{mL}$, respectively and minimum bactericidal concentration (MBC) ranged between $1.25-12.5 \mathrm{mg} / \mathrm{mL}$, for respectively against resistant $S$. aureus isolates using leaves methanol and aqueous $P$. guajava extracts (Anas et al., 2008). Indeed, MIC value was 10.3 and 7.3 $\mathrm{mg} / \mathrm{mL}$ against $S$. aureus and $E$. coli isolates, respectively. Whereas, it was $25 \mu \mathrm{g} / \mathrm{mL}$ against $S$. aureus and $0.78 \mu \mathrm{g} / \mathrm{mL}$ against both $E$. coli and B. subtilis pathogens using methanolic $P$. guajava leaf extracts (Dhiman et al., 2011). While, it was ranged between $0.125->0.250 \mathrm{mg} / \mathrm{mL}$ and between $0.062->0.250$ $\mathrm{mg} / \mathrm{mL}$ against the tested isolate with aqueous and methanolic extracts, respectively against $8 \mathrm{~S}$. aureus (MRSA) isolates with $P$. guajava stem bark aqueous and methanolic extracts (Esimone et al., 2012). Whereas, Saleh et al. (2015) reported that the estimated MIC values varied between 4 and $7.2 \mathrm{mg} / \mathrm{mL}$ for bacterial, whereas, it ranged between 14.5 and $37.3 \mathrm{mg} / \mathrm{mL}$ for fungal isolates. While, ethyl acetate had the lowest antimicrobial activity compared to the other tested solvents. Moreover, Taura et al. (2014) reported that MIC values for P. guajava leaves were recorded to be
250 and $500 \mu \mathrm{g} / \mathrm{mL}$ against Salmonella spp. and Pseudomonas spp. respectively at $45^{\circ} \mathrm{C}$, whereas, they recorded to be 500, 1000 and $1000 \mu \mathrm{g} / \mathrm{mL}$ against Klebsiella spp., Pseudomonas spp. and Salmonella spp. isolates, respectively at $60^{\circ} \mathrm{C}$. In the current study, MIC value was recorded to be 10.3 and $25 \mathrm{mg} / \mathrm{mL}$ against $S$. aureus and E. coli; whereas, it was 16.7 and $25 \mathrm{mg} / \mathrm{mL}$; while, it was 7 and $14.3 \mathrm{mg} / \mathrm{mL}$ against the two isolates using methanolic, ethanolic and hot water LE extracts.Whereas, Gitika and Kumar (2016) reported that the lowest MIC value was recorded to be 12.5 $\mathrm{mg} / \mathrm{mL}$ against $B$. subtilis, $M$. luteus, $S$. aureus and $E$. coli with methanolic leaves and with ethanolic leaves against $M$. luteus, and E. coli; and also with aqueous leaves against $M$. luteus isolate using $P$. guajava leaves

\section{Conclusion}

Antimicrobial activity of LE, SE, FE and TE $P$. guajava (methanol, ethanol, acetone, ethyl acetate and hot water) extracts was investigated against 8 bacterial isolates. Data presented herein showed that seeds (SE) and fruits (FE) extracts have no activity against all tested pathogens regardless tested solvent. Otherwise, ethyl acetate extract was the lowest potent against all tested isolates regardless studies plant fractions. Overall, methanolic LE and hot water $\mathrm{TE}<1 \mathrm{~cm}$ diameter extracts were the most potent against all tested microorganisms by showing the highest ZI and A.I value and lowest MIC values. The current investigation showed that $P$. guajava L. could use as an antibacterial agent with low cost and potential source in future researches. Further research however, on separately chemical components inhibitory activity of methanolic LE and hot water $\mathrm{TE}<1 \mathrm{~cm}$ extracts is requested.

\section{Acknowledgement}

We thank I. Othman (Director General of AECS) and N. Mir Ali (Head of Molecular Biology and Biotechnology Department in AECS) for their support, and also the Plant Biotechnology Group for technical assistance.

Conflict of Interest. The authors declare that there is no conflict of interest

\section{References}

Abdelrahim, S.I., Almagboul, A.Z.,Omer, M.E.A., Elegami, A. 2002 Antimicrobial activity of Psidium guajava L. Fitoterapia, 73: 713-715.

Ahmed, R.S., Yagoub, S.O. 2007. In vitro anti-microbial 
activity of Psidium guajava extracts. Research Journal of Microbiology, 2: 845-850.

Anas, K., Jayasree, P.R., Vijayakumar, T., ManishKumar, P.R. 2008. In vitro antibacterial activity of Psidium guajava Linn. leaf extract on clinical isolates of multidrug resistant Staphylococcus aureus. Indian Journal of Experimental Biology, 46: 41-46.

Biswas, B., Rogers, K., McLaughlin, F., Daniels, D., Yadav, A. 2013. Antimicrobial activities of leaf extracts of Guava (Psidium guajava L.) on two gram-negative and gram-positive bacteria. International Journal of Microbiology, 6: 136-141.

Dhiman, A., Nanda, A., Ahmad, S., Narasimhan, B. 2011. In vitro antimicrobial activity of methanolic leaf extract of Psidium guajava L. Journal of Pharmacy and Bioallied Sciences, 3: 226-229.

Egharevba, H.O., Iliya, I., Ibekwe, N., Abdullahi, M.S., Okwute, S.K., Okogun, J.I. 2010. Broad spectrum antimicrobial activity of Psidium guajava Linn. leaf. Nature and Science, 8: 43-50.

Elekwa, I., Okereke, S.C., Ekpo, B.O. 2009. Preliminary phytochemical and Antimicrobial investigations of the stem bark and leaves of Psidum guajava L. Journal of Medicinal Plant Research, 3: 045-048.

Esimone, C.O., Attama, A.A., Mundi, K.S., Ibekwe, N.N., Chah, K.F. 2012. Antimicrobial activity of Psidium guajava Linn. stem extracts against methicillin-resistant Staphylococcus aureus. African Journal of Biotechnology, 11: 15556-15559.

Farhana, J.A., Hossain M.F., Mowlah, A. 2017. Antibacterial effects of Guava (Psidium guajava L.) extracts against food borne pathogens. International Journal of Nutrition and Food Sciences, 6: 1-5.

Gitika, Kumar, M. 2016. Antibacterial activity of Psidium guajava L. leaves extracts against some Gramnegative bacteria. European Journal of Pharmaceutical and Medical Research, 3: 261-266.

Hassimotto, N.M., Genovese, M.I., Lajolo, F.M. 2005. Antioxidant activity of dietary fruits, vegetables, and commercial frozen fruit pulps. The Journal of Agricultural and Food Chemistry, 53: 2928-2935.

Ifeanyichukwu, I., Chika, E., Emmanuel, N., Anthonia, O., Ngozi, A., Agabus, N. 2015. Preliminary investigation of the antibacterial activity of Psidium guajava extracts. European Journal of Medicinal Plants, 7: 26-30.

Mahfuzul Hoque, MD., Bari, M.L., Inatsu, Y., Juneja,V.K., Kawamoto, S. 2007. Antibacterial activity of guava (Psidium guajava L.) and Neem (Azadirachta indica A. Juss.) extracts against foodborne pathogens and spoilage bacteria. Foodborne Pathogens and Disease, 4: 481-488.

Mailoa M., Mahendradatta, M., Laga, A., Djide, N., 2014. Antimicrobial activities of tannins extract from guava leaves (Psidium guajava L) on pathogens microbial. International Journal of Scientific and Technology Research, 3: 236-241.

Mani, A., Mishra, R., Thomas, G. 2011. Elucidation of diversity among Psidium species using morphological and SPAR methods. Journal of Phytology, 3: 53-61.

Nwinyi, O.C., Chinedu, N.S., Ajani, O.O. 2008. Evaluation of antibacterial activity of Psidium guajava and Gongronema latifolium. Journal of Medicinal Plant Research, 2: 189-192.

Ofodile, N.L., Nwakanma, N.M.C., Mordi, M., Ademolu, O., Ezimoke, I., Owoso, J. 2013. Genotoxic and antimicrobial studies of the leaves of Psidium guajava. Europian Asian journal of Bio Sciences, 7: 60-68.

Pandey, A. Shweta, C. 2012. Antibacterial properties of Psidium guajava leaves, fruits and stems against various pathogens. International Journal of Pharmaceutical Research and Development, 3: 15-24.

Rattanachaikunsopon, P., Phumkhachorn, P. 2010. Contents and antibacterial activity of flavonoids extracted from leaves of Psidium guajava. Journal of Medicinal Plant Research, 4: 393-396.

Saleh, B., Al-Halab, L., Al-Mariri, A. 2015. In vitro leaves and twigs antimicrobial properties of Psidium guajava L. (Myrtaceae). Herba Polonica, 61: 93 104.

Shruthi, S.D., Roshan, A., Timilsina, S.S., Sunita, S. 2013. A review on the medicinal plant Psidium guajava Linn (Myrtaceae). Journal of Drug Delivery and Therapeutics, 3: 162-168.

Taura, D.W., Yusha'u, M., Bello, U.A., Hassan, A., Saidu, J., Panda, T.W. 2014. Antibacterial activity of Psidium guajava in clinical isolates. Academia Journal of Microbiology Research, 2: 79-83. 